\title{
Reduction in staff smoking rates in North Coast Area Health Service, NSW, following the introduction of a smoke-free workplace policy
}

\author{
Gavin S. Dart ${ }^{\mathrm{A}}$, Eric K. van Beurden ${ }^{\mathrm{A}, \mathrm{B}}$, \\ Avigdor Zask $^{\mathrm{A}}$, Chalta Lord ${ }^{\mathrm{A}}$, Annie M. Kia ${ }^{\mathrm{A}}$ \\ and Ros Tokley \\ ${ }^{\mathrm{A}}$ Health Promotion, Population Health, Planning and Performance, \\ North Coast Area Health Service \\ ${ }^{\mathrm{B}}$ Corresponding author.Email: eric.vanbeurden@ncahs.health. \\ nsw.gov.au
}

\begin{abstract}
Aim: To evaluate changes in staff smoking rates following the implementation of Smoke Free Health Care, an innovative, changemanagement process that introduced a smoke-free workplace policy in the North Coast Area Health Service of NSW. Methods: Survey questionnaires were sent to all staff before and after the introduction of the policy. Return rates were $17.3 \%$ (690/ 3988 ) in 1999 and 25.4\% (2012/7921) in 2007. Chi-square tests and multivariate logistic regression analysis were used to determine differences. Results: Staff smoking rates decreased significantly from $22.3 \%$ to $11.8 \%(p<0.0001)$. Smoking rates in 1999 were not significantly different to the state population's ( $22.3 \%$ and $24.1 \%, p=0.3$ ), but were significantly different in 2007 (11.8\% and $20.1 \%$, $p<0.0001)$. Over a quarter $(27.6 \%)$ of staff who smoked when implementation began quit smoking; more than twice the rate before implementation $(12 \%, p<0.0001)$. Conclusion: These changes in staff smoking rates indicate the effectiveness of a comprehensive change-management approach to implementing smoke-free workplace policy.
\end{abstract}

Smoking harms nearly every organ of the body, causing many diseases and reducing the general health of smokers. ${ }^{1}$ Exposure to secondhand smoke can also cause premature death and disease in adults and children who do not smoke. ${ }^{2}$ Smoke-free workplace policies targeting smoking cessation have been introduced in a number of settings. ${ }^{3}$ Some have led to moderate decreases in staff smoking rates and cigarette consumption by employees who continue to smoke. ${ }^{4}$ A systematic review of 26 studies of smoke-free workplaces showed that totally smoke-free workplace policies had approximately twice the effect on reducing staff smoking rates (3.8\% reduction) as workplace policies that allowed smoking in some areas other than work areas. ${ }^{4}$ The World Health Organization recommends that all health care facilities be smoke-free and not permit smoking on the premises either indoors or outdoors. $^{5}$

In 1999 NSW Health directed all health services across the state to become totally smoke-free through the introduction of the NSW Smoke Free Workplace Policy. ${ }^{6}$ In response, the North Coast Area Health Service (NCAHS) developed a unique change-management process called Smoke Free Health Care and implemented it in all sites.

To our knowledge nothing has been published describing changes in staff smoking rates following the introduction of a smoke-free workplace policy across multiple sites in an Australian regional health service.

This paper presents an evaluation of changes in staff smoking and smoking cessation rates before and after implementation of Smoke Free Health Care.

\section{Methods}

\section{Study population}

NCAHS is a regional area health service in New South Wales (NSW), Australia. It employs approximately 8000 staff and services a population of more than 500000 people. The study involved cross-sectional health service staff smoking surveys conducted in 1999 and 2007. NCAHS consisted of two separate area health services in 1999: Northern Rivers and Mid North Coast (amalgamated in 2005). The pre-survey was conducted in the Northern Rivers Area Health Service only (3988 staff). The post-survey covered the whole of NCAHS (7921 staff) but included a question to identify those working in previous Northern Rivers Area Health Service sites. Data from statewide adult health surveys in 1998 and 2005 were used for comparison with health service staff smoking rates. 7,8 


\section{Intervention}

NCAHS implemented a systematic and innovative organisational change-management process termed Smoke Free Health Care between 2002 and 2007 that introduced a policy banning smoking in indoor and outdoor areas of NCAHS sites. ${ }^{6}$ Smoke Free Health Care was multi-strategic; along with policy directives, it incorporated mandatory workforce education, humour and improved clinical intervention for nicotine dependent inpatients with smoking cessation and harm minimisation support for staff who smoked. ${ }^{9}$ All Smoke Free Health Care strategies were strongly supported at the executive level. ${ }^{9}$ Smoke Free Health Care was implemented within the context of other population-wide environmental and behavioural initiatives aimed at reducing smoking rates.

\section{Survey design}

Two cross-sectional surveys of area health service staff, one administered in 1999 and the other in 2007, were used to collect smoking data. The first survey was conducted in 1999 before the implementation of Smoke Free Health Care. It established a baseline staff smoking rate and measured interest in smoking cessation. The follow-up survey was conducted in 2007 after the implementation of Smoke Free Health Care. This survey assessed pre and post Smoke Free Health Care staff smoking rates, smoking cessation rates and the perceived success or otherwise of Smoke Free Health Care (SFHC). Survey instruments were developed by a reference group with expertise in tobacco control and public health evaluation (Box 1). Ethics approvals for the 1999 and 2007 surveys were granted by the Human Research and Ethics Committees of the Northern Rivers Area Health Service and NCAHS respectively.

\section{Data collection and management}

The surveys were in Teleform format (a layout that allows handwritten and tick box responses to be detected by an electronic scanner and exported to a computer database) and distributed to all staff with their payslips. ${ }^{10}$ Return rates were $17.3 \%(690 / 3988)$ in 1999 and 25.4\% (2012/ 7921 ) in 2007. Returned surveys were manually checked and scanned. Data were imported into a database, coded and imported into SAS (version 8.02, SAS Institute, Cary, $\mathrm{NC}, \mathrm{USA}$ ) for final cleaning and analysis.

\section{Data analysis}

SAS was used to derive frequencies and means. Confidence intervals for proportions were calculated using the method of Fleiss. ${ }^{11}$ Chi-square tests were used for bivariate analyses. Multivariate logistic regression included pre, post, age and gender as predictors of smoking cessation rates to determine age-adjusted pre to post changes. ${ }^{11}$ Estimates of sampling bias were determined by comparing age by gender and smoking status of the survey sample with relevant workforce and NSW Health survey data. ${ }^{7,8,12}$

Area health service smoking and cessation rates were compared with NSW population rates obtained from NSW Health Survey data. ${ }^{7,8}$ There were no published data for the years our surveys were administered. Therefore, 1999 survey data were compared with 1998 state population data and the 2007 survey data were compared with the 2005 state population data.

NCAHS sites were progressed to smoke-free status sequentially between 2003 and 2007. Analyses to compare pre and post smoking cessation rates were therefore sitespecific and involved all sites surveyed at baseline. The date respondents indicated they had quit smoking was linked to the date their site went smoke-free, which occurred after an intensive 'Smoke-Free Transition' intervention. The proportion of staff that quit smoking after their site went smoke-free was compared to the proportion of staff that quit smoking, in an equal time period, before the site went smoke-free. For example, where a site went smoke-free in September 2006, the number of staff who reported quitting smoking between September 2006 and the 2007 follow-up survey was compared to the number of staff who reported quitting smoking within the same number of months before September 2006.

\section{Results}

The follow-up sample was older than the baseline sample with $67.1 \%$ of respondents aged over 40 years in 1999 and $77.5 \%$ in $2007\left(\chi^{2}=100.85, \mathrm{df}=1, p<0.0001\right)$. Both surveys had a ratio of approximately $3: 1$ females to males $\left(\chi^{2}=0.42, \mathrm{df}=1, p=0.5162\right)$. This is consistent with the general health workforce. ${ }^{4}$

\section{Box 1. Information collected from the 1999 and 2007 Smoke Free Health Care surveys}

- Demographics: gender, age, workplace, employment history $(1999,2007)$

- Smoking status: daily, occasional, quit, never $(1999,2007)$

- Interest in smoking cessation and readiness to use nicotine replacement therapy (1999)

- If ceased, when: month and year (2007)

- Reasons for cessation: medical advice, influence of colleagues, health, television, billboards and graphic packet warnings, cost of cigarettes, influence of friends and/or family, my worksite going totally smoke-free, and other (2007)

- Perceived success or otherwise of Smoke Free Health Care (2007) 


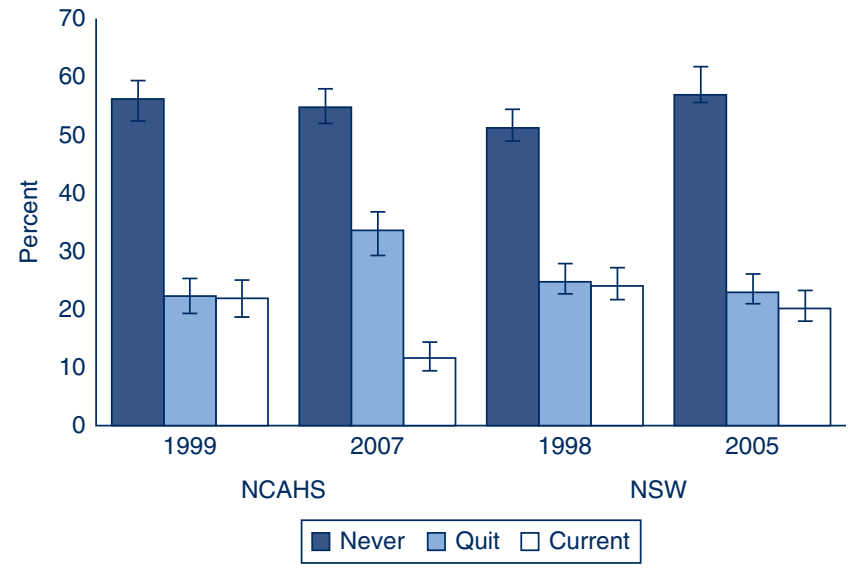

Figure 1. Rates and confidence intervals of 'never smoked', 'quit smoking' and 'current smoker' in North Coast Area Health Service staff compared to NSW population.

\section{NCAHS staff smoking rate compared to NSW population}

Comparisons were made between smoking rates in NCAHS staff samples and the NSW population. ${ }^{7,8}$ In 1999, smoking rates in the staff sample and the NSW population were not statistically different $(22.3 \%$ compared to $24.8 \% ; \chi^{2}=1.01, \mathrm{df}=1, p=0.3156$ ). In 2007 , smoking rates in the staff sample were significantly lower than the NSW population (11.8\% compared to $22.2 \%$; $\left.\chi^{2}=69.80, \mathrm{df}=1, p<0.0001\right)($ Figure 1).

\section{Smoking status: pre and post SFHC implementation}

There were significant decreases in the current staff smoking rate for females from $20.3 \%$ to $11.1 \%$ and for males from $24.6 \%$ to $13.6 \%$. Staff smoking cessation rates increased substantially between the pre and post surveys. For females, the rate increased from $21.3 \%$ to $34.1 \%$ and for males from $24.6 \%$ to $32.7 \%$. This contrasted with reduced smoking cessation in the NSW population (females: $21.2 \%$ to $20.1 \%$; males: $28.4 \%$ to $25.5 \%$ ).

The regression model revealed that pre to post SFHC changes in smoking cessation rates were highly significant even after taking into account the effects of age and gender $\left(\beta_{\text {pre-post }}=-0.9412, \quad\right.$ se $\left.=0.1465, p<0.0001\right)$. Smoking cessation rates increased with age $\left(\beta_{\text {age }}=\right.$ -4742 , se $=0.0744, p<0.0001)$ but were unaffected by gender $\left(\beta_{\text {gender }}=-0.0915\right.$, se $\left.=0.1613, p<0.5703\right)$. Parameter estimates were used to generate predicted adjusted smoking cessation rates and changes in cessation rates for selected age by gender cohorts. Age cohorts with sufficient numbers at either end of the scale were chosen to represent these changes. Predicted changes in ex-smokers ranged from $16 \%(66 \%$ to $82 \%)$ in more senior female staff to $20 \%$ (27\% to $47 \%$ ) for younger males (Figure 2).

Over a quarter (27.6\%) of staff who smoked when their site went smoke-free subsequently quit smoking (Figure 3). This reduction is significant and more than double that for

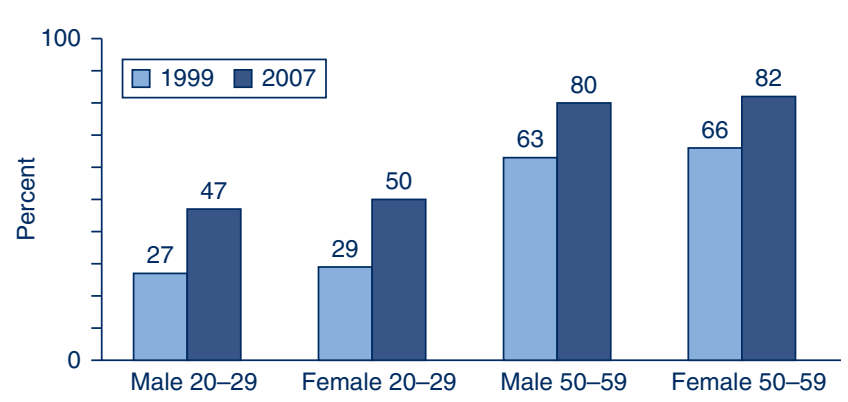

Figure 2. Staff smoking cessation rates in North Coast Area Health Service pre and post implementation of Smoke Free Health Care, by age and gender.


Figure 3. North Coast Area Health Service staff smoking profile pre and post implementation of Smoke Free Health Care (of respondents who were smoking when Smoke Free Health Care commenced at their site).

the same time period before SFHC $\left(12.0 \%, \chi^{2}=27.35\right.$, $\mathrm{df}=1, p<0.0001)$. For those who quit smoking and gave a reason $(n=192)$, the four main reasons were: health (32.8\%); family/friends (19.8\%); cost (17.7\%); and worksite going smoke-free (10.4\%).

\section{Perceived success of SFHC}

Approximately half (51.2\%) of the 2007 sample considered SFHC to have 'worked fairly or very well', while the remainder considered it to have "worked OK but still with difficulties' or to have 'not worked at all well'. Respondents who were still smoking were less positive with only $38.2 \%$ responding 'fairly or very well'.

\section{Discussion}

A $10 \%$ reduction in staff smoking rates occurred after the implementation of SFHC. This is considerably larger than the mean of 3.8\% reported by Fichtenberg and Glantz in their systematic review. ${ }^{4}$ The apparent success was achieved in spite of initial resistance from staff at many 
sites. While some respondents expressed dissatisfaction with certain aspects of SFHC, most considered it had been successfully implemented.

A potential limitation of this study is the cross-sectional survey design and low response rates. These limit generalisability and the extent to which we can attribute the observed decrease in smoking to the intervention alone. However, this design was deemed most feasible in a sequential, service-wide initiative within the broader context of statewide policy implementation. Comparison with statewide population smoking rates strengthened the design. As an additional measure of intervention efficacy, the inclusion of smoking cessation rates, in comparative time periods before and after SFHC implementation, provided another source of triangulation.

Self-selection may have introduced some bias in relation to the main outcomes. However, comparisons between the survey sample and workforce demographics, and also NSW Health surveys, suggest this bias was minimal.

The possible influence of the ageing of the workforce was precluded by the adjustment for age and gender effects in the multivariate analysis. This indicated that initial estimates, using age-adjusted survey data, resulted in underestimation of the full impact on staff smoking rates. The fact that approximately half of the 2007 survey respondents considered SFHC had worked 'fairly' or 'very well' and the other half considered that it had worked 'not at all well' or 'OK but with some difficulties' further suggests that if there was self-selection bias in either direction on this scale, it was not excessive.

The degree of change in staff smoking rates evident over the period of SFHC implementation provides support for smoke-free workplace policies that remove all designated smoking areas, include a staff education component and support staff smoking cessation through a comprehensive organisational change-management process. SFHC implementers recommend that wherever smoke-free policy exemptions exist, staff health and safety risk assessments should be undertaken because the evidence suggests that maintaining designated smoking areas is likely to contribute to continued smoking by staff. This was acknowledged in an internal Phillip Morris memo in 1992 which referred to a body of company research that reported: 'Total prohibition of smoking in the workplace strongly affects industry volume.... Milder workplace restrictions, such as smoking only in designated areas, have much less impact on quitting rates.' 13

\section{Conclusion}

Decreases in staff smoking rates and increases in smoking cessation rates evident from the pre and post SFHC implementation surveys were large and significant when compared to secular trends. This intervention appears to have been more effective than other interventions reported in the literature. The results support existing evidence for the effectiveness of smoke-free workplace interventions that simultaneously implement environmental and behavioural strategies.

\section{Acknowledgments}

We would like to thank Mark Mitchell, Christine Sullivan and Uta Dietrich for questionnaire design comments, Gail Bennett for Teleform development, Kate Jackson for data collection and Ian Smith for workforce data support.

\section{References}

1. CDC Office on Smoking \& Health. National Center for Chronic Disease Prevention \& Health Promotion - CDC. The health consequences of smoking. A report of the Surgeon General. Atlanta: CDC's Office on Smoking \& Health; 2004.

2. U.S. Department of Health \& Human Services. The health consequences of involuntary exposure to tobacco smoke: A report of the Surgeon General. U.S. Department of Health \& Human Services, Centers for Disease Control and Prevention, Coordinating Center for Health Promotion, Office of Smoking and Health; 2006.

3. Moher M, Hey K, Lancaster T. Workplace interventions for smoking cessation Cochrane Database Syst Rev 2005; (Issue 2): CD003440.

4. Fichtenberg CM, Glantz SA. Effect of smoke-free workplaces on smoking behaviour: systematic review. BMJ 2002; 325: 188-94. doi: $10.1136 /$ bmj.325.7357.188

5. Raw M, Anderson P, Batra A, Dubois G, Harrington P, Hirsch A et al. WHO Europe evidence based recommendations on the treatment of tobacco dependence. Tob Control 2002; 11(1): 44-6. doi:10.1136/tc.11.1.44

6. Health NSW. Smoke Free Workplace Policy. Sydney: NSW Department of Health; 1999.

7. Public Health Division, NSW Department of Health. Report on the 1997 and 1998 NSW Health Surveys. Available from: www. health.nsw.gov.au/PublicHealth/surveys/hsa/9798/hsindex.htm (Cited November 2007.)

8. Centre for Epidemiology and Research. 2005 Report on Adult Health from the New South Wales Population Health Survey. Sydney: NSW Department of Health; 2006.

9. Kia AM, van Beurden EK, Dart GS, Barrack CM, Mitchell MD. Smoke Free Health Care: an organisational change to increase effective intervention for tobacco. NS W Public Health Bull 2008; 19(3-4): 60-4. doi:10.1071/NB07115

10. Autonomy. Autonomy TeleForm: Product Brief. 2009.

11. Fleiss JL. Statistical Methods for Rates and Proportions. 2nd ed. New York: John Wiley and Sons; 1981.

12. North Coast Area Health Service. Business objects database (Sep07) 2007.

13. Philip Morris USA. Impact of workplace restrictions on consumption and incidence (inter-office correspondence). 1992. Available from: http://legacy.library.ucsf.edu/tid/ rpo98e00/pdf (Cited 24 January 2008.) 